\title{
Toy Model for Uncommon Spin-orbit-driven Spin-Torque Terms
}

\author{
Charles Paillard ${ }^{1,2}$ * Raymond Walter ${ }^{2,3,4}$, Surendra \\ Singh $^{2}$, Brahim Dkhil ${ }^{1}$, and L. Bellaiche ${ }^{2,3}$ \\ 1 Laboratoire SPMS, CentraleSupelec/CNRS UMR8580, \\ Université Paris-Saclay, 92295 Châtenay-Malabry Cedex, France \\ 2 Physics Department, University of Arkansas, Fayetteville, Arkansas 72701, USA \\ 3 Institute for Nanoscience and Engineering, \\ University of Arkansas, Fayetteville, Arkansas 72701, USA and \\ ${ }^{4}$ Mathematics Department, University of Arkansas, Fayetteville, Arkansas 72701, USA
}

(Dated: April 2, 2017)

\begin{abstract}
A toy model combining the Angular MagnetoElectric (AME) coupling Hamitonian [Mondal et al, Phys. Rev. B 92, 100402(R) (2015)] with long-range magnetic dipolar interactions is used to investigate spin-torque phenomena in a magnetic spin valve. It is found that such model (1) gives rise to spin-torque expressions that are analogous in form to those of the common spin-transfer torques; but also (2) predicts additional spin-torque terms, which are generated by an electrical current oriented along unconventional, in-plane directions. The magnitude of the AME induced terms is estimated and the conditions under which they may contribute significantly are explored.
\end{abstract}




\section{INTRODUCTION}

We consider a toy model to investigate spin-torque effects in a simple and physicallytransparent fashion, as a tribute to the numerous phenomenological models that Dr. Ekhard Salje developed throughout his career to extract the essence of complex physical phenomena, e.g., the field of surface relaxation ${ }^{122}$ and phase transitions ${ }^{3}$.

Independently predicted in 1996 by Slonczewski ${ }^{4}$ and Berger ${ }^{\sqrt{5}}$, spin-transfer torque (STT) is a phenomenon in which a spin polarized current can exert a torque on magnetic moments (the spin torque). Spin-torque has grown increasingly popular ${ }^{\sqrt[6]{8}}$ in recent years since it holds the promise of applications in spintronics and beyond. For instance, maintaining the gyrotropic motion of a magnetic vortex in heterojunction nanopillars via spin-torque is an elegant solution for designing new nanoscale microwave emitters ${ }^{910}$. Similarly, spin-torque allows one to move domain walls ${ }^{11} 13$, resulting in the control of the resistance state $e^{14}$ and this may very well find applications in the so-called memristors ${ }^{8}$. Spin-torque is thus one of the finest examples of magnetoelectric effect.

Spin-torque, as described in the STT model, is considered to be an interfacial effect in which reflection and transmission of the incident wavefunction at the interface cause spin filtering at the interface, leading to spin precession of the transmitted wave and spin rotation of the reflected wavefunction ${ }^{715}$. No a priori spin-orbit coupling is necessary for spin-torque to take place: spin filtering is generated by the mismatch of the Fermi surfaces at the interface ${ }^{15}$, and spin precession, for instance, depends critically on the splitting of the majority spin and minority spin bands. However, spin-orbit coupling can enhance or even induce such splitting through, for instance, the Rashba ${ }^{16}$ and Dresselhaus ${ }^{17}$ effects. Indeed, the so-called spin-orbit torques based on spin Hall and Rashba-like effects, are believed to be of significant importance in heterostructures containing heavy metal layers (such as those made of $\mathrm { Pt } \longdiv { 1 8 } \sqrt { 2 0 }$.

Interestingly, a pioneering study ${ }^{21}$ predicted that novel spin-torques can directly originate from spin-orbit couplings rather than being a consequence of spin current transfer. Technically, this study combined the effect of magnetic exchange interactions with the "traditional" spin-orbit coupling Hamiltonian ${ }^{22}$ :

$$
\mathcal{H}_{S O}=-\frac{e \hbar}{4 m^{2} c^{2}}[\boldsymbol{E} \times \boldsymbol{p}] \cdot \boldsymbol{\sigma},
$$

where $\boldsymbol{E}$ is the electric field, $\boldsymbol{p}$ the momentum operator, $\boldsymbol{\sigma}$ the vector of Pauli matrices, $\hbar$ 
the reduced Planck constant, $m$ the mass of the electron, and $c$ the velocity of light.

The spin-orbit coupling Hamiltonian of Eq. (1) is not gauge invariant under the local $U(1)$ symmetry group, that is, the change of wavefunction $\psi$ by a phase factor $\psi^{\prime}=e^{i \theta} \psi$ does not leave $\mathcal{H}_{S O}$ invariant for non-constant and non-uniform phase $\theta$. This gauge invariance, which is a fundamental symmetry of quantum mechanics, can be restored by adding an appropriate spin-dependent Hamiltonian to $\mathcal{H}_{S O}$. This additional spin-coupling, which goes beyond the traditional spin-orbit coupling represented by $\mathcal{H}_{S O}$, arises naturally when the nonrelativistic limit of Dirac equation is considered in a gauge invariant manner. It has been reconsidered ${ }^{23}$ recently after appearing to have been mostly overlooked for nearly 60 years 2425 . It has already been shown to be relevant to the spin-current mode ${ }^{26}$ in multiferroics; the anomalous Hall27, anisotropic magneto-resistance and planar Hall 28 , and inverse Rashba-Edelstein 29 effects in magnetic materials; and the inverse Faraday effect ${ }^{23}$. Furthermore, such coupling seems to be at the origin of the difference in magnetization dynamics observed in pump-probe experiments with different relative orientation of the polarization of the pump and probe beams ${ }^{30131}$. This spin-coupling Hamiltonian has been called the Angular MagnetoElectric (AME) Hamiltonian ${ }^{23}$ and is given by:

$$
\mathcal{H}_{A M E}=\frac{e^{2} \hbar}{4 m^{2} c^{2}} \boldsymbol{\sigma} \cdot[\boldsymbol{E} \times \boldsymbol{A}],
$$

where $\boldsymbol{A}$ is the vector potential. An elegant recent work has suggested that the AME Hamiltonian by itself is capable of producing a spin-torque ${ }^{32}$, which was called the optical spin-torque. While the works of Refs. ${ }^{21 / 32}$ are remarkable and novel, they involve detailed and complex derivations required to accurately treat the spin-orbit-driven spin-torques arising from relativistic quantum mechanical effects. Here, we consider a simple toy model, involving the AME coupling and dipole-dipole interactions, and explore the possibility of novel spinorbit-driven spin-torques arising within a physically-transparent framework.

\section{AME-INDUCED MAGNETIC FIELD AND RESULTING SPIN TORQUE}

Let us first emphasize that Eq. (2) implies that the AME coupling is responsible for the emergence of an effective magnetic field $\boldsymbol{B}_{A M E}$ given by:

$$
\boldsymbol{B}_{A M E}=\frac{e^{2} \hbar}{4 m^{2} c^{2} g \mu_{B}}[\boldsymbol{E} \times \boldsymbol{A}],
$$


where $g$ is the Landé factor of the electron and $\mu_{B}$ the Bohr magneton. This effective magnetic field has already been shown to lead to a relativistic correction to the so-called Inverse Faraday Effect, in which a circularly polarized light wave can generate a helicity dependent magnetic field, which can be used to control magnetization 23133 .

Note that, in Eqs. (1)-(3), the electric field $\boldsymbol{E}(\boldsymbol{r})$ is a priori the total electric field, and can thus be decomposed into a microscopic part, $\boldsymbol{E}_{l o c}(\boldsymbol{r})$, and an applied external electric field, $\boldsymbol{E}_{\text {ext }}$, as:

$$
\boldsymbol{E}(\boldsymbol{r})=\boldsymbol{E}_{l o c}(\boldsymbol{r})+\boldsymbol{E}_{\text {ext }} .
$$

In the case of a non-vanishing external electric field, there is a response of the solid, which in the limit of small fields can be approximated to be linear. As a result, $\boldsymbol{E}_{l o c}(\boldsymbol{r})=\boldsymbol{E}_{l o c}^{0}(\boldsymbol{r})+$ $\gamma(\boldsymbol{r}) . \boldsymbol{E}_{\text {ext }}$, where $\boldsymbol{E}_{\text {loc }}^{0}(\boldsymbol{r})$ is the microscopic field in the absence of external electric field, and $\gamma(\boldsymbol{r})$ (which is in general a tensor) characterizes the response of the microscopic electric field to the applied field. Since the microscopic field $\boldsymbol{E}_{l o c}^{0}(\boldsymbol{r})$ averages to zero in the unit cell, for vector potential $\boldsymbol{A}$ varying slowly enough, the contribution of the microscopic part of the AME-induced effective magnetic field is negligible, and is left out of the following discussion. In addition, in order to simplify the discussion, we will consider $\gamma$ to be a positionindependent scalar and uniform, that is, a cubic, homogeneous and isotropic material is considered. We will thus take the following form of $\boldsymbol{B}_{A M E}$,

$$
\boldsymbol{B}_{A M E}=(1+\gamma) \frac{e^{2} \hbar}{4 m^{2} c^{2} g \mu_{B}}\left[\boldsymbol{E}_{\text {ext }} \times \boldsymbol{A}\right]=a\left[\boldsymbol{E}_{\text {ext }} \times \boldsymbol{A}\right],
$$

with $a=(1+\gamma) \frac{e^{2} \hbar}{4 m^{2} c^{2} g \mu_{B}}$ characterizing the strength of the AME coupling.

From the form of the effective AME magnetic field, it is clear that such a field will exist for non-vanishing external electric field and potential vector, and thereby exert a torque on any magnetic moment $\boldsymbol{\mu}$ in the material, as stated in Ref. ${ }^{32}$. Indeed, such an effective field can be included in the Landau-Lifshitz-Gilbert (LLG) equation" ${ }^{143435}$ governing the time evolution of $\boldsymbol{\mu}$,

$$
\frac{d \boldsymbol{\mu}}{d t}=-\frac{\gamma_{0}}{\mu_{0}} \boldsymbol{\mu} \times\left(\boldsymbol{B}_{\text {other }}+\boldsymbol{B}_{A M E}\right)+\frac{\alpha}{\mu_{0}} \hat{\boldsymbol{\mu}} \times\left[\boldsymbol{\mu} \times\left(\boldsymbol{B}_{\text {other }}+\boldsymbol{B}_{A M \boldsymbol{E}}\right)\right],
$$

where $\gamma_{0}=\frac{g \mu_{0} \mu_{B}}{\hbar}$ is the electronic gyromagnetic factor, $\mu_{0}$ is the vacuum permeability, $\alpha$ is the damping constant, and $\hat{\boldsymbol{\mu}}$ is the unit vector along the magnetic moment $\boldsymbol{\mu}$. $\boldsymbol{B}_{\text {other }}$ represents the magnetic field arising from sources other than the AME Hamiltonian. 
For instance, $\boldsymbol{B}_{\text {other }}$ can arise from anisotropy, exchange, or even possibly DzyaloshinskiiMoriya ${ }^{36 / 37}$ and dipolar interactions. The total effective magnetic field is therefore $\boldsymbol{B}_{\boldsymbol{t o t}, \boldsymbol{e f f}}=$ $\boldsymbol{B}_{\text {other }}+\boldsymbol{B}_{\boldsymbol{A M E}}$. The first term of Eq. (6) is the usual torque acting on a magnetic moment, causing it to precess around the axis of the total effective magnetic field, while the second term is the usual damping term favoring the magnetic moment $\boldsymbol{\mu}$ to be aligned

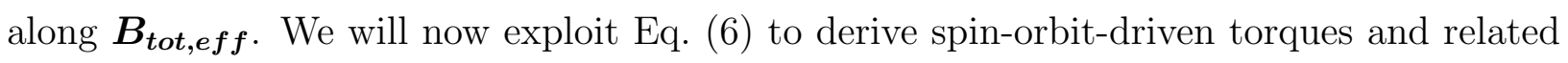
effects associated with magnetic dipolar interactions. For simplicity (i.e., to make the subsequent derivations rather straightforward), these latter interactions will correspond to the long-range dipolar ones.

\section{DIPOLE-DIPOLE INTERACTIONS AND AME INDUCED SPIN-TORQUE}

\section{A. Geometry of the system and dipolar vector potential}

Let us consider a typical spin-valve geometry depicted in Fig. 1. Two magnetic layers are separated along the z-axis by a non-magnetic layer, denoted as $N M$. The first magnetic fixed layer, $M_{1}$, exhibits a uniform magnetization $\boldsymbol{M}$ oriented along a fixed direction (say, the $y$ axis), while the second free magnetic layer, $M_{2}$, possesses magnetic dipoles, $\boldsymbol{\mu}$, that are free to rotate. The whole system is taken to have infinite dimensions in the $x$ and $y$ directions, and is subjected to external electric field, $\boldsymbol{E}_{\boldsymbol{e x} \boldsymbol{t}}$, that we will assume to be uniform without further assumptions about its direction.

Consider now the dipolar vector potential created by an elemental magnetic moment $\boldsymbol{M} \delta V$ of $M_{1}$ located at $\boldsymbol{r}_{\boldsymbol{m}}$ at the location $\boldsymbol{r}$ of a magnetic moment $\boldsymbol{\mu}$ of layer $M_{2}$ :

$$
d \boldsymbol{A}_{\text {dip }}=\frac{\mu_{0}}{4 \pi} \frac{\boldsymbol{M} \delta V \times\left(\boldsymbol{r}-\boldsymbol{r}_{\boldsymbol{m}}\right)}{\left|\boldsymbol{r}-\boldsymbol{r}_{\boldsymbol{m}}\right|^{3}}
$$

The magnetization of the bottom layer is assumed to be fixed, and therefore the dipolar potential is not time dependent. Summing over all dipoles of the magnetic layer $M_{1}$ of thickness $d$ then yields

$$
\boldsymbol{A}_{\text {dip }}=\frac{1}{2} \mu_{0} d(M \hat{\boldsymbol{y}} \times \hat{\boldsymbol{z}}) .
$$

where $\boldsymbol{y}$ is the unit vector along $\boldsymbol{M}$ and $\boldsymbol{z}$ is the unit vector normal to the interface.

It is interesting to note that the associated magnetic field $\boldsymbol{\nabla} \times \boldsymbol{A}_{\boldsymbol{d i p}}=\mathbf{0}$ vanishes outside the infinitely extended layer $M_{1}$ with in-plane uniform magnetization ${ }^{38}$ but $\boldsymbol{A}_{\boldsymbol{d i p}}$ of Eq. (8) 


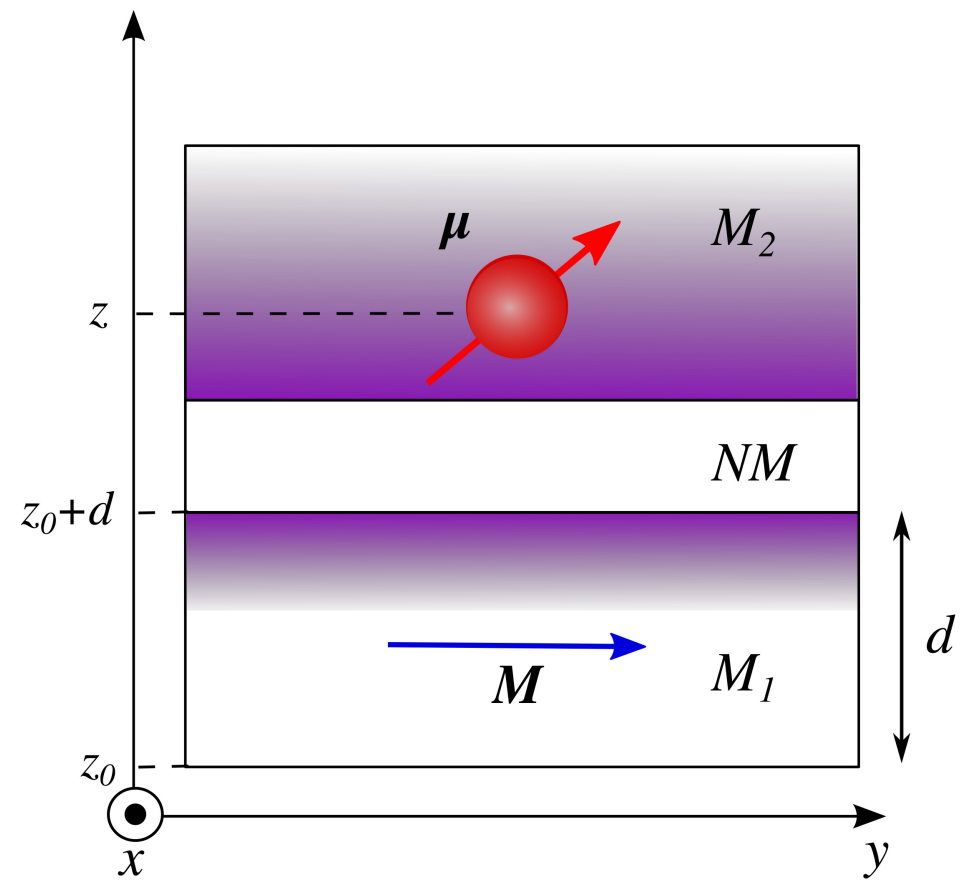

FIG. 1. Schematics of spin torque geometry. The uniformly magnetized material $M_{2}$ of thickness $d$ and magnetization $\boldsymbol{M}$ along the $y$-axis is separated from the magnetic layer $M_{2}$ by a non-magnetic layer $N M$ along the $z$-axis.

does not. This vector potential, when combined with Eq. (5), thus leads to a spin-orbitdriven AME effective magnetic field given by

$$
\boldsymbol{B}_{\boldsymbol{A M E}, \boldsymbol{d i p}}=\frac{a \mu_{0} d}{2 g \mu_{B}}\left(\boldsymbol{E}_{\text {ext }} \cdot \hat{\boldsymbol{z}}\right) M \hat{\boldsymbol{y}}-\frac{a \mu_{0} d}{2 g \mu_{B}}\left(\boldsymbol{E}_{\text {ext }} \cdot M \hat{\boldsymbol{y}}\right) \hat{\boldsymbol{z}}
$$

This effective magnetic field will generate a torque on a spin magnetic moment, which we now calculate. 


\section{B. Induced Spin Torques}

Choosing the anisotropy field $\boldsymbol{B}_{\text {other }}$ along $\boldsymbol{M}$ as in Refs. $\frac{439}{\text {, }}$, and using the expression for $\boldsymbol{B}_{A M E}$ from Eq. (9) in Eq. (6) we obtain:

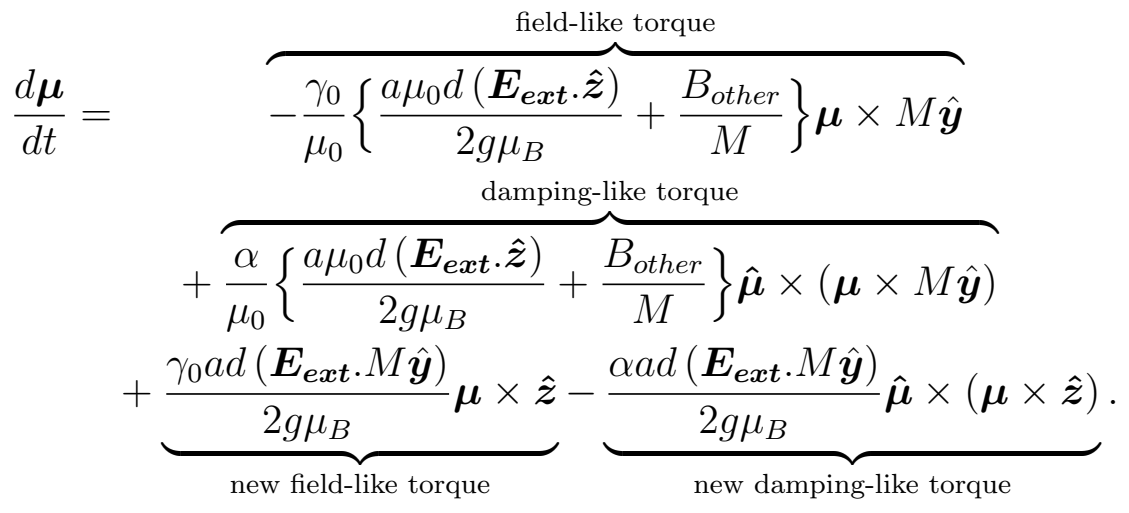

Interestingly, the first two terms of this equation have precisely the same analytical form as the known field and damping torques ${ }^{15}$ since they depend on $\boldsymbol{\mu} \times M \hat{\boldsymbol{y}}$ and $\hat{\boldsymbol{\mu}} \times(\boldsymbol{\mu} \times M \hat{\boldsymbol{y}})$, respectively, which demonstrates that our rather simple model can capture the analytical forms of known, complex effects. These two first terms reach their full strength when the current is flowing in direction $\hat{\boldsymbol{z}}$ (i.e., $\boldsymbol{E}_{\boldsymbol{e x t}}$ is parallel to $\hat{\boldsymbol{z}}$ ). This geometry is similar to the typical spin-torque geometry for maximizing the field and damping-spin torques ${ }^{15}$. The first two terms of Eq. 10 can be considered as spin-orbit-driven contributions to the field and damping spin torques since Eq. (2) originates from the relativistic Dirac Hamiltonian 23. Interestingly, each of these two terms is directly proportional to the sum $\frac{a \mu_{0} d\left(\boldsymbol{E}_{\text {ext }} . \hat{\boldsymbol{z}}\right)}{2 g \mu_{B}}+\frac{B_{\text {other }}}{M}$, which can be made to vanish for a critical value of the external field given by $\boldsymbol{E}_{\boldsymbol{e x t}} \cdot \hat{\boldsymbol{z}}=$ $-\frac{2 B_{\text {other }} g \mu_{B}}{a \mu_{0} M d}$, at which point the AME effective field exactly compensates the anisotropy field. Beyond this critical field, both the field-like torque and damping-like torque in Eq. (10) change sign, which results in reversing the magnetic moment $\boldsymbol{\mu}$. Such a critical field is usually expressed in terms of critical current $\boldsymbol{j}_{\boldsymbol{c}, \boldsymbol{d i p}}$, which in our case can be written

$$
\boldsymbol{j}_{\boldsymbol{c r}, \boldsymbol{d i p}}=-\frac{2 \sigma_{z z} B_{\text {other }} g \mu_{B}}{a \mu_{0} M d} \hat{\boldsymbol{z}}
$$

where $\sigma_{z z}$ is the longitudinal conductivity.

Let us now estimate the strength of this critical current density for, e.g., Cobalt material (for simplicity, we assume $M_{1}$ and $M_{2}$ are both made of Co, as in $\mathrm{Co} / \mathrm{Cu} / \mathrm{Co}$ heterostruc-

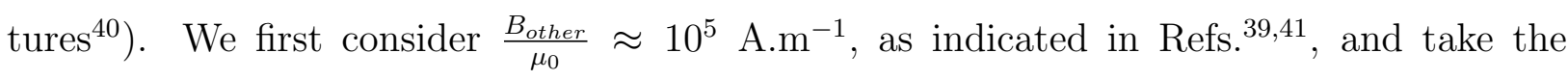
conductivity of Cobalt to be $\sigma_{z z}=1.54 \times 10^{7}{\mathrm{~S} . \mathrm{m}^{-1}}$ as in Ref.42]. We also take $M$, the 
magnitude of the magnetization of Co, to be equal to $1.42 \times 10^{6} \mathrm{~A}^{-\mathrm{m}^{-1}}$, as in Refs. $\frac{39140}{\text {. We }}$ then estimate the $a$ coefficient by recalling that the anomalous Hall effect (AHE) can be described by the AME Hamiltonian ${ }^{27}$, which allows us to write the transverse conductivity associated with AHE as $\sigma_{A H E}=\frac{a}{2 g \mu_{B}} M$. Taking again $M$ to be $1.42 \times 10^{6} \mathrm{~A} . \mathrm{m}^{-1}$ and $\sigma_{A H E}$

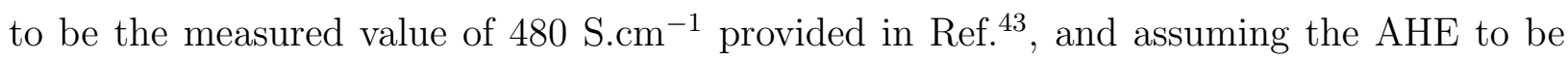
fully derived from the AME coupling, gives a coefficient $a$ equal to $1.3 \times 10^{-24}{\mathrm{~S} . \mathrm{m}^{2}}^{2}$. We further assume, as realized in $\mathrm{Co} / \mathrm{Cu} / \mathrm{Co}$ nanopillars $\frac{40}{4}$, that the thickness $d$ of the bottom $\mathrm{M}_{1}$ magnetic layer is equal to $10 \mathrm{~nm}$. As a result, the critical current density given by Eq. (11) can be estimated to be $1.3 \times 10^{14} \mathrm{~A} . \mathrm{cm}^{-2}$ for $j_{c r, d i p}$. This value is two to three orders of

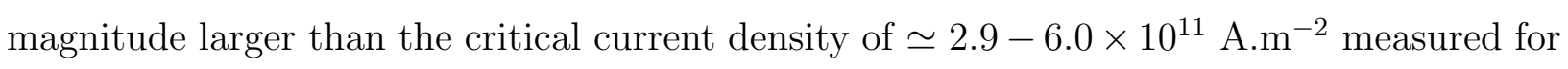
moderate magnetic fields $(0.6 \mathrm{kOe})$ in Ref. ${ }^{40}$, implying that the first two terms of Eq. 10 should be considered as perturbative corrections to the known "traditional" spin-torques. Note, however, that $j_{c r, \text { dip }}$ is inversely proportional to the thickness of the $M_{1}$ layer. For instance, it becomes equal to $1.3 \times 10^{13} \mathrm{~A} . \mathrm{cm}^{-2}$ for $d=100 \mathrm{~nm}$ and therefore should have a more pronounced effect for a thicker fixed layer (this dependence of $j_{c r, d i p}$ on $1 / d$ arises from the assumption of $M_{1}$ having infinitely large in-plane dimensions in the derivation of Eq. (8), and would obviously break down for very thick layers.).

We now shift our attention to the third and fourth terms in Eq. (10), which differ qualitatively from the conventional spin-transfer torque model. For instance, these two terms are maximal when the external electric field is applied along the (in-plane) direction of the uniform magnetization $\boldsymbol{M}$ of $M_{1}$ - rather than along the normal to the interface $\hat{\boldsymbol{z}}$ as in typical STT experiments 5 . In that case, the third term favors a precession of $\boldsymbol{\mu}$ around $\hat{\boldsymbol{z}}$ while the fourth term favors a rotation of $\boldsymbol{\mu}$ towards or away from $\hat{\boldsymbol{z}}$ depending on the sign of the dot product between the applied electric field and $\boldsymbol{M}$. These third and fourth terms, therefore, characterize unconventional spin-orbit-driven field- and damping-torques ${ }^{15}$, respectively, for which experimental confirmation would require applying an electric field having a non-vanishing component along the magnetization of the $M_{1}$ bottom layer, according to Eq. 10). Interestingly, Ref.21 also predicted that an applied electric field having a non-vanishing projection along the direction of $\boldsymbol{M}$ can indeed generate a torque, via the combined effect of exchange interaction and a Rashba (spin-orbit-driven) coupling 16 . However, this latter torque was found to be parallel to $\boldsymbol{\mu} \times M \hat{\boldsymbol{y}}$ rather than to $\boldsymbol{\mu} \times \hat{\boldsymbol{z}}$ as in our case. 
Moreover, and, as already noted, the vector potential of Eq. (8) gives rise to a magnetic field $\boldsymbol{\nabla} \times \boldsymbol{A}_{\text {dip }}=0$ outside the magnetic layer $M_{1}$. As a result, the existence the AME induced spin-torques of Eq. (10) depends on nonzero vector potential (even though the magnetic field itself is zero) and can have observable effects. This situation is akin to the Aharonov-Bohm effect ${ }^{45}$ and reminds us that the electromagnetic potentials are fundamental in quantum mechanics rather than the fields.

\section{Gauge invariance}

Let us now add the gradient of a scalar function to $\boldsymbol{A}_{\text {dip }}$, which does not affect this vanishing magnetic field. In particular, one can choose the gradient of this function to be precisely the opposite of the right-hand side of Eq. (8). This would then result in a new $\boldsymbol{A}_{\text {dip }}$ that will now fully vanish - which will also completely annihilate the effective AME magnetic field of Eq. (9)! However, this change of $\boldsymbol{A}_{\text {dip }}$ corresponds, in fact, to a change of gauge, and, as a result, the traditional spin-orbit Hamiltonian of Eq. (1) will acquire a new quantity that has to be added to the momentum, with this quantity being precisely the right-hand side of Eq. (8). With this change of gauge, the spin-orbit spin torques of Eq. (10) will therefore still exist but will now arise from the traditional spin-orbit Hamiltonian rather than the AME one. These considerations indicate the importance of the AME Hamiltonian both from the fundamental as well as practical points of views and that it is not always possible to identify a physical effect as arising from the AME or the traditional SOC Hamiltonian. This is because the sum of the traditional spin-orbit coupling and AME Hamiltonians is gauge invariant rather than each individual Hamiltonian 32|46.

\section{SUMMARY}

In summary, we employed a simple toy model combining the AME coupling with the long-range dipolar vector potential, which leads to the prediction of (i) spin-orbit-driven spin-torques having the same analytical form as the classical STT but also of (ii) uncommon spin torques. It is important to emphasize that the spin torques discussed here are fundamentally different from the spin-orbit effects reported in Refs. $\stackrel{20 \mid 47}{ }$ for which spin-orbit was used to create a spin-polarized current (via the spin Hall effect) that induced spin-transfer 
torque. Here the spin-orbit effects directly drive the spin torques, while they are merely STT enhancers in Refs. ${ }^{20147}$ with the spin-torque originating from the usual transfer of the spin-current. We hope that this simple toy model will stimulate further (and perhaps justify more elaborate) theoretical developments in the field of spin-orbit-driven spin-torques. For instance, one may also be interested to consider the combination of the AME coupling with magnetic exchange interactions to determine if it can also lead to spin-orbit-driven spin torques having the same analytical forms as those indicated in Eq. (10) (but with smaller critical currents since magnetic exchanges are stronger in magnitude than long-range dipolar interactions). Of course, an ultimate check of our predictions has to be done by performing experiments, such as confirming the existence of the uncommon spin torques (i.e., for which the electrical current is applied along in-plane directions) via measurements in systems possessing strong-spin-orbit couplings.

There authors thank Vincent Cros and Albert Fert for insightful and detailed comments on the work and manuscript. C.P. thanks a public grant overseen by the French National Research Agency (ANR) as part of the "Investissements d'Avenir" program (reference: ANR10-LABX-0035, Labex NanoSaclay) and Department of Energy, Office of Basic Energy Sciences, under contract ER-46612, for personal support during its visit to Fayetteville, AR. R.W. acknowledges this material is based upon work supported by the National Science Foundation Graduate Research Fellowship Program under Grant No. DGE-0957325, the University of Arkansas Graduate School Distinguished Doctoral Fellowship, and the Department of Energy Office of Basic Energy Sciences under contract ER-46612. L.B. thanks the support of the ARO grant W911NF-12-10085.

* charles.paillard@centralesupelec.fr

1 B. Houchmanzadeh, J. Lajzerowicz and E. Salje, Phase Transitions 38, 77 (1992).

2 B. Houchmanzadeh, J. Lajzerowicz and E. Salje, J. Phys.: Condens. Matter 4, 9779 (1992).

3 B. Houchmanzadeh, J. Lajzerowicz and E. Salje, J. Phys.: Condens. Matter 3, 5163 (1991)

4 J. Slonczewski, J. Mag. Magn. Mat. 159, L1 (1996)

${ }^{5}$ L. Berger, Phys. Rev.B 54, 9353 (1996)

6 M. D. Stiles and J. Miltat, Spin Dynamics in Confined Magnetic Structures III, pp. 225-308, 
Springer Berlin Heidelberg (2006)

7 D. Ralph and M. D. Stiles, J. Mag. Magn. Mat. 320, 1190 (2008)

8 N. Locatelli, V. Cros and J. Grollier, Nat. Mat. 13, 11 (2014)

9 S. I. Kiselev, J. C. Sankey, I. N. Krivorotov, N. C. Emley, R. J. Schoelkopf, R. A. Buhrman and D. C. Ralph, Nature 425, 380 (2003)

10 A. Dussaux, B. Georges, J. Grollier, V. Cros, A. V. Khvalkovskiy, A. Fukushima, M. Konoto, H. Kubota, K. Yakushiji, S. Yuasa, K. A. Zvezdin, K. Ando and A. Fert, Nat. Comm. 1, 8 (2010)

11 S. P. Parkin, M. Hayashi and L. Thomas, Science 320, 190 (2008)

12 K.-S. Ryu, L. Thomas, S.-H. Yang and S. P. Parkin, Nat. Nano. 8, 527 (2013)

13 A. V. Khvalkovskiy, V. Cros, D. Apalkov, V. Nikitin, M. Krounbi, K. A. Zvezdin, A. Anane, J. Grollier and A. Fert, Phys. Rev. B 87, 020402 (2013)

14 J. Grollier, P. Boulenc, V. Cros, A. Hamzić, A. Vaurès, A. Fert and G. Faini, Appl. Phys. Lett. 83, $509(2003)$

15 M. D. Stiles and A. Zangwill, Phys. Rev. B 66, 014407 (2002)

16 Y. Bychkov, E. Rashba, J. Phys. C 17, 6039 (1984)

17 G. Dresselhaus, Phys. Rev. 100, 580 (1955)

18 P. M. Haney, H. W. Lee, K. J. Lee, A. Manchon, and M. D. Stiles, Phys. Rev. B 88, 214417 (2013)

19 F. Freimuth, S. Blügel and Y. Mokrousov, Phys. Rev. B 90, 174423 (2014)

20 L. Liu, C.-F. Pai, Y. Li, H. W. Tseng, D. C. Ralph and R. A. Buhrman, Science 336, 555 (2012)

21 A. Manchon and S. Zhang, Phys. Rev. B 79, 094422 (2009)

22 P. Strange, Relativistic Quantum Mechanics with applications in condensed matter and atomic physics, Cambridge University Press, New York (1998)

23 R. Mondal, M. Berritta, C. Paillard, S. Singh, B.Dkhil, P. M. Oppeneer and L. Bellaiche, Phys. Rev. B 92, 100402(R) (2015)

24 L. L. Foldy, S. A. Wouthuysen, Phys. Rev. 78, 29 (1950)

25 K. M. Case, Phys. Rev. 95, 1323 (1954)

26 A. Raeliarijaona, S. Singh, H. Fu, L. Bellaiche, Phys. Rev. Lett. 110, 137205 (2013)

27 L. Bellaiche, W. Ren and S. Singh, Phys. Rev. B 88, 161102(R) (2013) 
R. Walter, M. Viret, S. Singh and L. Bellaiche, J. Phys.: Cond. Mat. 26, 432201 (2014)

29 S. Bhattacharjee, S. Singh, D. Wang, M. Viret and L. Bellaiche, J. Phys.: Cond. Mat. 26, $315008(2014)$

30 J.-Y. Bigot, M. Vomir and E. Beaurepaire, Nat. Phys. 5, 515 (2009).

31 R. Mondal, M. Berritta and P. M. Oppenneer, arXiv:1701.05376v2 (2017).

32 R. Mondal, M. Berritta, P. M. Oppeneer, Phys. Rev. B 94, 144419 (2016).

33 M. Berritta, R. Mondal, K. Carva, P. M. Oppeneer, Phys. Rev. Lett. 117, 137203 (2016)

34 L. Landau and E. Lifshitz, Phys. Z. Sowjetunion 8, 153 (1935)

35 T. Gilbert, IEEE Transactions on Magnetics 40, 3443 (2004)

36 I. Dzyaloshinskii, J. Phys. Chem. Sol. 4, 241 (1958)

37 T. Moriya, Phys. Rev. 249, 1949 (1960)

38 R. Allenspach, J. Magn. Magn. Mat. 129, 160 (1994)

39 J. Grollier, Renversement d'aimantation par injection d'un courant polarisé en spin, $\mathrm{PhD}$ thesis, Université Pierre et Marie Curie - Paris VI, October 2003

40 J. A. Katine, F. J. Albert, R. A. Buhrman, E. B. Myers, and D.C. Ralph, Phys. Rev. Lett. 84, $3149(2000)$

41 J. Z. Sun, Phys. Rev. B 62, 570 (2000)

42 G. V. Samsonov, Handbook of the Physical Properties of the Elements, Chapter 4, p. 316, Springer (2012)

43 X. Wang, D. Vanderbilt, J. R. Yates, I. Souza, Phys. Rev. B 76, 195109 (2007)

44 Note that in the absence of a response of the solid, i.e. $\gamma=0, a \approx 9 \times 10^{-31} \mathrm{~S}^{2} \mathrm{~m}^{2}$, and thus the critical current is even larger, $j_{c r, d i p} \approx 1.8 \times 10^{20} \mathrm{~A} \cdot \mathrm{cm}^{-2}$.

45 Gordon Baym, Lectures on Quantum Mechanics (Benjamin Cummings, Reading, MA, 1969), pp 77.

46 C. Paillard, R. Mondal, M. Berritta, B. Dkhil, S. Singh, P. M. Oppeneer, L. Bellaiche, SPIE Optics and Photonics Proceedings (2016).

47 L. Liu, T. Moriyama, D. C. Ralph, R. A. Buhrman, Phys. Rev. Lett. 106, 036601 (2011) 